\title{
Complete excision of the jejunum and vessels for the treatment of esophageal cancer
}

\section{Running head: The treatment of esophageal cancer}

Hexiao Tang ${ }^{1 \&} \mathrm{MD}$, PhD, Lecai Xiong ${ }^{1 \&} \mathrm{MD}$, Yuquan Bai ${ }^{1} \mathrm{MD}$, Li Zhang ${ }^{1} \mathrm{MD}, \mathrm{PhD}$, Minglin Zhu ${ }^{1}$ MD, PhD, Shaoping Zhu ${ }^{1}$ MD, PhD, Gaofeng Pan ${ }^{1}$ MD, PhD, Xuefeng Zhou ${ }^{1}$ MD, PhD, Ming Xu ${ }^{1}$ MD, PhD, Jinping Zhao ${ }^{1 *}$ MD, Ph.D

${ }^{1}$ Department of Thoracic and Cardiovascular Surgery, Zhongnan Hospital of Wuhan University, Wuhan 430071, China

${ }^{\&}$ Co-first author. They make the same contribution to this article.

* Correspondence to: Jinping Zhao, MD, Ph.D, Director, the Department of Thoracic and Cardiovascular Surgery, Zhongnan Hospital of Wuhan University, Wuhan 430071, China. Telephone number: 086-13808699392.E-mail: ZhaoJPthx@126.com 


\begin{abstract}
Herein, we reported a case of complete excision of the jejunum for the treatment of esophageal cancer. A 62-year-old male was admitted with complaints of "difficulty in eating for one month and chest pain for 10 days". For complete gastrectomy and colonic polyposis, we chose the jejunum. We completely excised the jejunum and its vessels, anastomosed to the esophageal tumor resection, with no significant complications. This case showed that complete excision of the jejunum with bowel and vessels is an alternative surgical method for the treatment of esophageal cancer with free bowel shortage and no torsion of blood vessels.
\end{abstract}

\title{
Keywords
}

Esophageal cancer; Residual stomach; Colon polyps; Jejunum

\section{Introduction}

Esophageal cancer is one of the most common causes of cancer-related deaths worldwide, and surgical treatment is critical for healing and improving survival of patients. The standard approach of radical surgery is to remove the esophageal tumor lesions and systemic lymph node dissection, and replace and reconstruct the upper gastrointestinal tract by autologous tissue transplantation. The order of choice for esophageal replacement organs are stomach, colon and jejunum [1]. This case focuses on the application of jejunum replacement in radical esophagectomy.

\section{Case Description}

A 62-year-old male was admitted to our hospital with complaints of "difficulty in 
eating for one month and chest pain for 10 days". The endoscopy and colonoscopy performed 10 days before surgery, showed esophageal lesions $30-35 \mathrm{~cm}$ to incisors; stomach change to Billroth II, slight congestion on the anastomotic mucosa (Figure 1a), and normal jejunum. The digestive tract imaging on November 7, 2015, showed the changes after esophageal radical surgery, with no abnormalities by small bowel angiography (Figure 1c, d). The patient underwent esophageal radical surgery under general anesthesia on October 26, 2015 (Figure 2). Postoperative pathological examination indicated $\mathrm{T}_{1} \mathrm{~N}_{0} \mathrm{M}_{0}$. The patient was successfully discharged in December 2015 after successfully completing six courses of chemotherapy post-surgery, and followed-up for 1.5 years.

The surgical steps for complete excision of the jejunum with bowel and vessels in the treatment of esophageal cancer were as follows (Figure 2): (1) By the fifth intercostal lateral of left thoracic incision into the chest, we carefully released the esophageal traction; removed the lymph node under the carina and the lower esophagus; excised the esophagus from the top of the pleura and diaphragm on the level; the mushroom head was placed in the cavity for jejunum followed by esophageal anastomosis. (2) After administration of $4000 \mathrm{u}$ intravenous heparin, $30 \mathrm{~cm}$ of the jejunum with bowel and vessels was completely excised, followed by $5 \mathrm{~cm}$ to the gastric jejunum anastomosis. (3) The alternate jejunum arteries and descending aorta were anastomosed through discontinuous open venous pedicle, and the jejunum vein and azygos vein were anastomosed after satisfactory blood flow. We anastomosed two sides of the jejunum to the esophagus by stapler, then anastomosed the abdominal distal jejunum and the stomach wall by stapler. (4) Wound hemostasis was strictly maintained, followed by placement of thoracic cavity drainage tube, and closure of the abdomen. 


\section{Discussion}

Stomach and colon are the most commonly used alternative organs for total gastrectomy [2]. The jejunum has good compatibility with the esophagus, but has medium firmness, fragile blood circulation and limited free length, and is therefore, used only when the stomach and colon are unusable $[3,4]$. In this case, since the patient had a complete gastrectomy, colonic polyposis and new esophageal lesions $30-35 \mathrm{~cm}$ to the incisors, the only viable option was to anastomose the jejunum artery and descending aorta, which ensured satisfactory venous blood flow. Then, the jejunum vein and odd veins were anastomosed. The bowel color was bright red, with good intestinal peristalsis.

\section{Summary}

(1) We completely excised the jejunum and its blood vessels, reduced the wound with the length of the free bowel, the risk of vascular and intestinal torsion and the associated complications. (2) The method of jejunum artery connection to descending aorta and jejunum vein connection to azygos vein ensured adequate blood supply after anastomosis, narrowed the distance to facilitate the operation and decreased the anastomosis time, which were conducive to postoperative recovery. This case was successful, and more cases are required in the future for statistical analysis and collecting the treatment of related complication as medical evidence.

\section{Conflict of interest}

The authors declare no conflict of interest.

\section{Footnote}


Informed consent: Written informed consent was obtained from the patient for publication of this case report and accompanying images.

\section{References}

1. Citing a journal article:Zhang, Z., Y. Guo, C. Liang, et al. Free jejunum interposition as salvage surgery after cervical esophagus injury. J Thorac Dis 2016; E513-6.

2. Citing a journal article:Hirahara, N., T. Tanaka, S. Yano, et al. Reconstruction of the Gastrointestinal Tract by Hemi-Double Stapling Method for the Esophagus and Jejunum Using EEA OrVil in Laparoscopic Total Gastrectomy and Proximal Gastrectomy. Surgical Laparoscopy Endoscopy \& Percutaneous Techniques 2011; E11E15.

3. Citing a journal article:Briel, J.W., A.P. Tamhankar, J.A. Hagen, et al. Prevalence and risk factors for ischemia, leak, and stricture of esophageal anastomosis: gastric pullup versus colon interposition. J Am Coll Surg 2004; 536-41; discussion 541-2.

4. Citing a journal article:Urschel, J.D. Late dysphagia after presternal colon interposition. Dysphagia 1996; 75-7. 
Figures

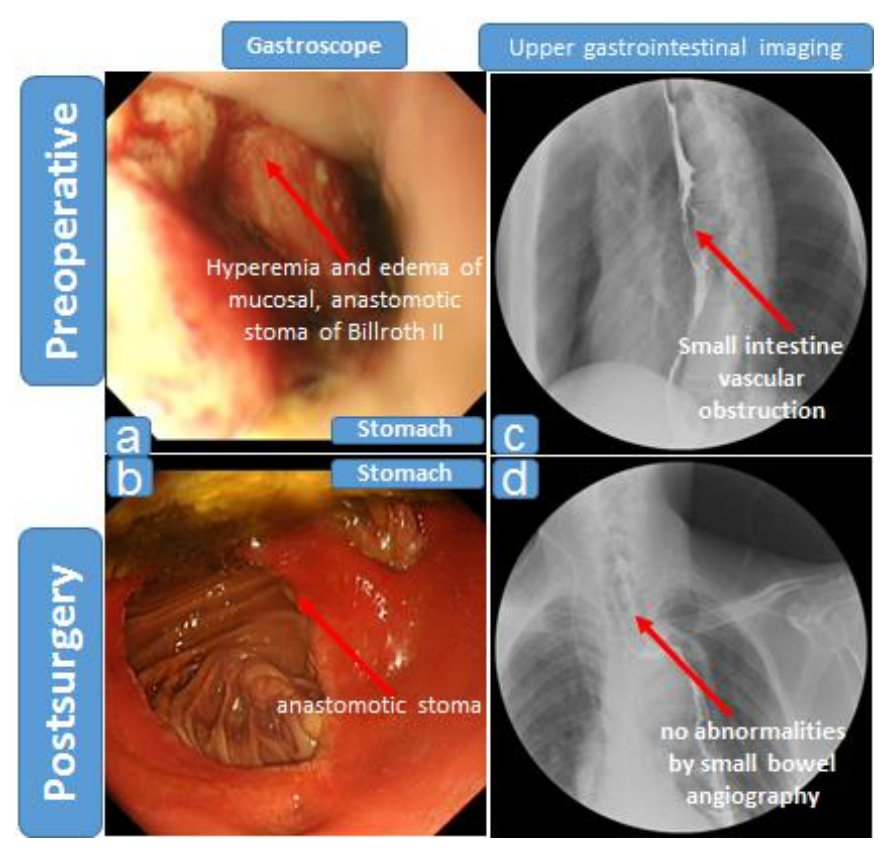

Figure. 1 Comparison of examination results between preoperative and postoperative Anastomotic stomach of Billroth II (a), anastomotic of jejunum and stomach (b), and changes of preoperative and postoperative in small bowel vascular changes (c, d).
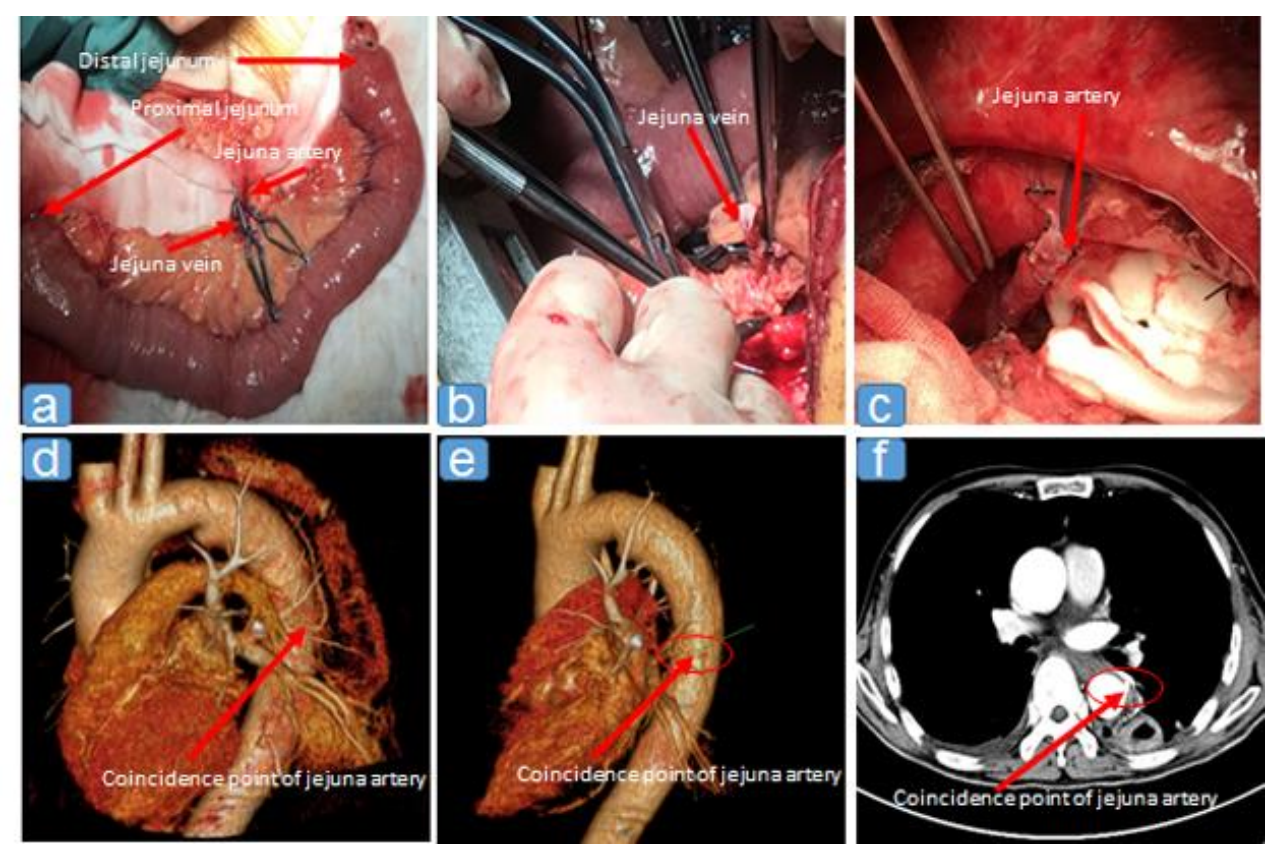

Figure. 2 Surgical vascular anastomosis and the results of validation

Completely broken off the bowel and blood vessels of jejunum (a); Anastomosis the 
jejunum vein (b); Anastomosis the jejunum artery (c). The results of imaging for jejunum artery anastomosis point, the anastomosis point of jejuna artery in arterial three - dimensional imaging (d, e) and aortic enhancement CT (f). 